\title{
A case of pulmonary alveolar microlithiasis with an intragenetic deletion in SLC34A2 detected by a genome-wide SNP study
}

\author{
Y Ishihara, ${ }^{1}$ K Hagiwara, ${ }^{2}$ K Zen, ${ }^{3}$ Huqun, ${ }^{2}$ Y Hosokawa, ${ }^{4}$ A Natsuhara ${ }^{1}$
}

${ }^{1}$ Department of Respiratory Medicine, Omihachiman Community Medical Center, Omihachiman, Japan;

${ }^{2}$ Department of Respiratory Medicine, Saitama Medical University, Saitama, Japan;

${ }^{3}$ Department of Cardiovascular

Medicine, Omihachiman

Community Medical Center,

Omihachiman, Japan;

${ }^{4}$ Department of Pathology,

Omihachiman Community

Medical Center, Omihachiman, Japan

Correspondence to:

Dr K Hagiwara, Department of Respiratory Medicine, Saitama

Medical University, 38

Morohongo, Moroyama,

Saitama 350-0495, Japan;

hagiwark@saitama-med.ac.jp

Received 11 June 2008 Accepted 16 October 2008

\begin{abstract}
A case of pulmonary alveolar microlithiasis occurring in an inbred family is presented. A genome-wide analysis of the patient's genomic DNA using a high-density single nucleotide polymorphism (SNP) array revealed a small intragenetic mutation at SLC34A2. The results suggest that the high-density SNP array has the power to identify a recessive disease gene(s) even in the analysis of only a single inbred patient.
\end{abstract}

Inbreeding increases the chance for a mutant gene to become homozygous in the offspring and thus for autosomal recessive diseases to develop. ${ }^{1}$ The increased homozygosity is attributed to the appearance of chromosomal regions called autosomal segments, ${ }^{2}$ where both strands of homologous chromosomes are identical by descent (ie, derived from a single chromosome of a single ancestor). In inbred children affected by an autosomal recessive disease, the causative gene is very likely to be in the autozygous segments. ${ }^{34}$ Recent high-density single nucleotide polymorphism (SNP) arrays are able to determine both of the SNP genotypes genome-wide and the copy number of each chromosomal location. A patient with pulmonary alveolar microlithiasis was investigated using the array which revealed a small intragenetic deletion in the SLC34A2 gene, the gene considered to be the cause of the disease.

\section{CASE REPORT}

A 56-year-old woman was admitted to hospital with progressive dyspnoea, severe systemic oedema and a weight increase from $45 \mathrm{~kg}$ to $60 \mathrm{~kg}$. She had been notified for the first time in her 30s that her chest radiograph was abnormal although, at that time, no subjective symptoms were present. A physical examination showed blood pressure $128 / 60 \mathrm{~mm} \mathrm{Hg}$, pulse rate $84 \mathrm{bpm}$ and arterial oxygen saturation $\left(\mathrm{SpO}_{2}\right)$ measured with a pulse oximeter of $90 \%$. Her pulmonary second heart sound was accentuated. Laboratory test results revealed increased serum levels of C-reactive protein $(2.3 \mathrm{mg} / \mathrm{dl})$, B-type natriuretic peptide $(1256 \mathrm{pg} / \mathrm{ml})$ and sialylated carbonhydrate antigen KL-6 (583 U/ml). A chest radiograph revealed numerous micronodules throughout the entire lung field and obscured the cardiac shadow (fig 1A). A CT scan of the chest showed consolidation and diffuse ground-glass opacities in both lungs, accompanied by dilated bronchi (fig 1B, upper panel). A marked enlargement of the right heart, retention of pleural and pericardial effusions and calcification involving the mediastinal and interlobular pleura and the pericardium were also observed (fig 1B, lower panel). The ECG revealed a right bundle branch block accompanied by a tall $R$ wave in $a V_{R}$ and $V_{1}$, deep $S$ and a small $r$ in $\mathrm{I}$ and $a V_{L}$ and a persistent $S$ or $\mathrm{s}$ in the left precardial leads. These findings suggested right ventricular hypertrophy. Spirometry showed restrictive ventilatory disturbances (vital capacity $51.6 \%$, forced expiratory volume in $1 \mathrm{~s} 93.1 \%$ ). Transthoracic echocardiography revealed a dilated right heart with tricuspid regurgitation (fig 1C, upper panel) where the right ventricle-right atrium pressure gradient was $81 \mathrm{~mm} \mathrm{Hg}$ and the peak tricupsid regurgitation jet speed was $4.5 \mathrm{~m} / \mathrm{s}$ (fig 1C, lower panel). Right heart catheterisation confirmed a systolic pulmonary artery pressure of $109 \mathrm{~mm} \mathrm{Hg}$. Transbronchial lung biopsy revealed calcospherites with a lamellar appearance (fig 1D, upper panel) that were positive for von Kossa stain (fig 1D, lower panel). These calcospherites were surrounded by many macrophages and multinucleated giant cells. The final diagnosis was pulmonary alveolar microlithiasis (PAM) with right-sided heart failure.

\section{Genome-wide SNP study}

A genome-wide SNP study was performed using an SNP Array 6.0 (Affymetrix, USA) which genotypes 906600 SNPs and determines the copy number of the chromosomal sequences at 946000 chromosomal locations. ${ }^{5}$ Autozygous segments were predicted from the genotyping data (fig 1E). All genes contained in the segments are homozygous and therefore recessive traits may reveal themselves in the phenotype. SLC34A2, a causative gene for PAM, was located in an autozygous segment on chromosome 4. The copy number data showed that the sequence at probe CN_1062321 was absent, suggesting that the segment it locates was homozygously deleted (fig 1F). A detailed PCR analysis confirmed a $5.5 \mathrm{~kb}$ long homozygous deletion that involved exons 2-6 of the SLC34A2 gene and thus caused PAM in this patient.

\section{DISCUSSION}

PAM is an autosomal recessive disease with more than 500 patients reported worldwide ${ }^{67}$ and with the mutation of SLC34A2 identified as the cause. ${ }^{89}$ The whole genome analysis showed the power of a recently developed SNP genotyping platform. If it is assumed that SLC34A2 was not known to be the 

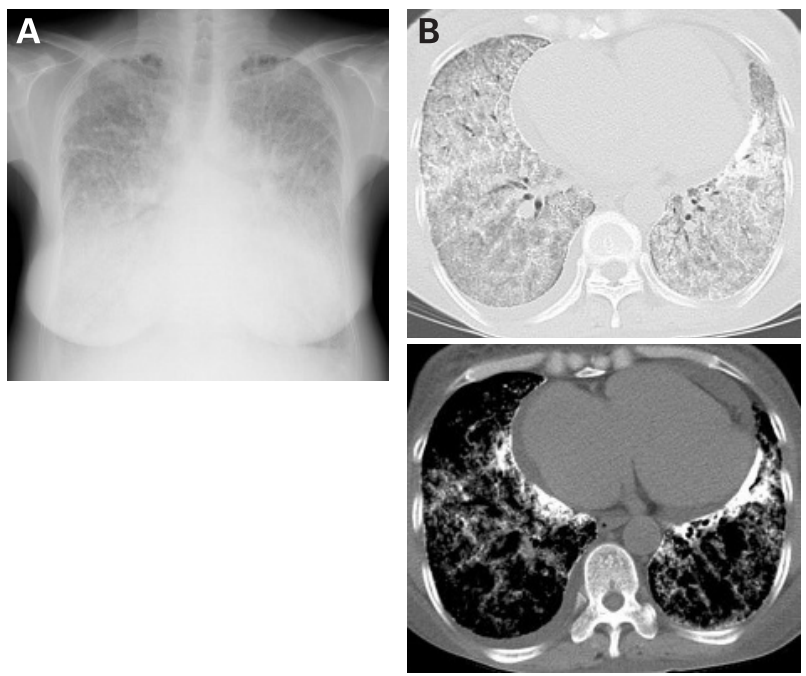

$\mathbf{E}$

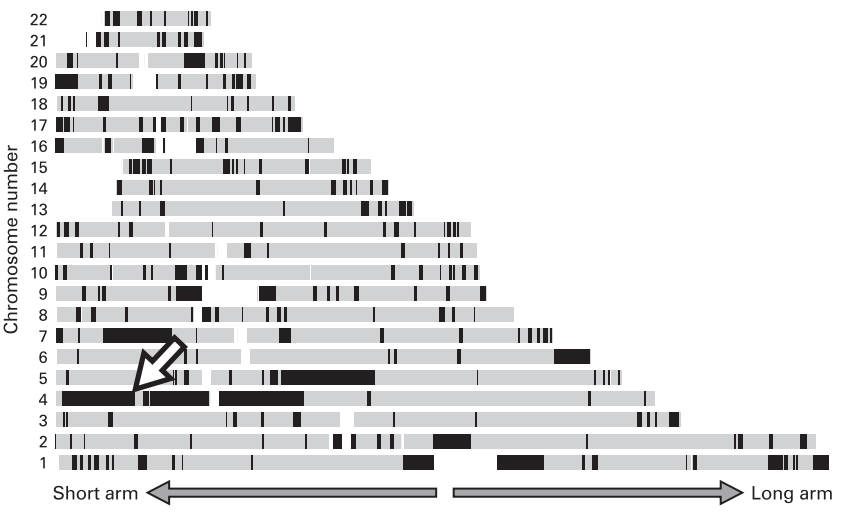

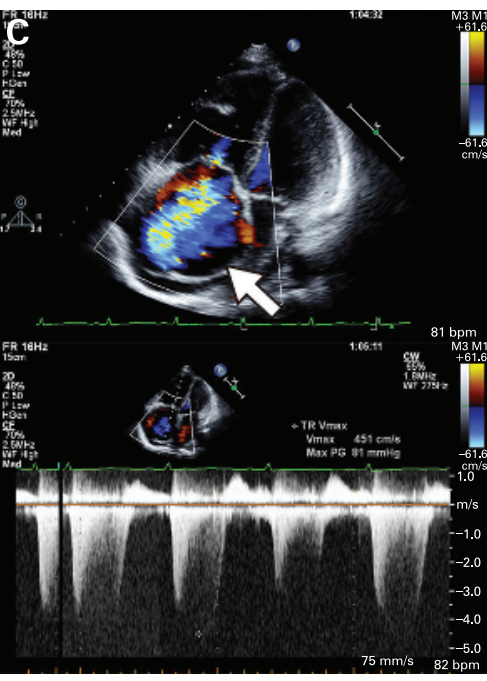

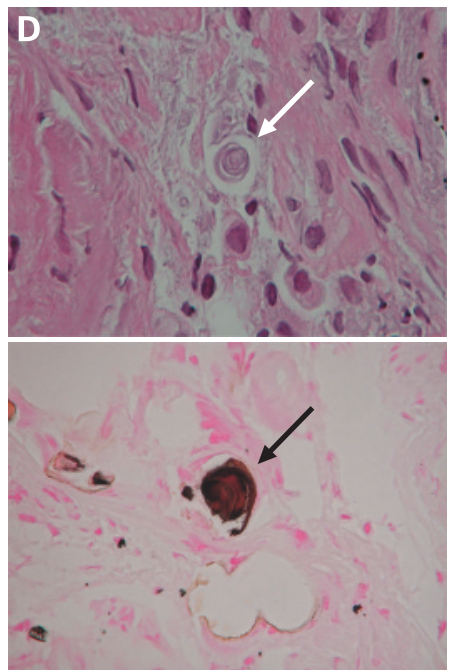

$\mathbf{F}$

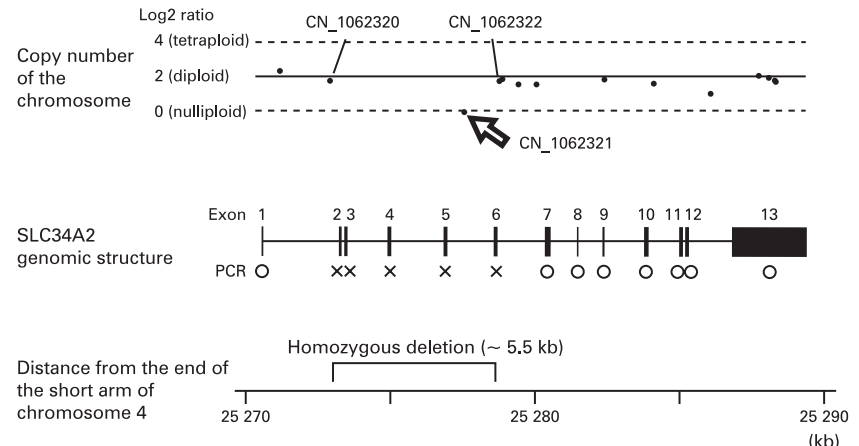

Figure 1 (A) Chest radiograph showing numerous micronodules throughout the entire lung field obscuring the cardiac shadow. This characteristic image is known as a "snow storm" appearance. (B) Upper panel: Lung window image of CT scan of the chest showing consolidation and diffuse ground-glass opacities in both lungs and dilated bronchi. Lower panel: Mediastinal window image showing marked enlargement of the right heart and mild retention of pleural and pericardial effusions accompanied by calcification of the mediastinal and interlobular pleura as well as the pericardium. (C) Upper panel: Colour transthoracic echocardiography Doppler image showing dilated right heart and severe tricuspid regurgitation (white arrow). Lower panel: Continuous wave Doppler image that estimated the right ventricular-right atrial pressure gradient and peak speed of the tricupsid regurgitation jet. (D) Upper panel: Thin section $(400 \times)$ of the decalcified lung tissue stained with haematoxylin and eosin showing a lamellar calcospherite (white arrow). Lower panel: The calcospherite is positive for von Kossa stain (black arrow). (E) Predicted autozygous segments (black bands) overlaid on the illustration of chromosomes (grey bands). SLC34A2 (indicated by a white arrow) is located in an autozygous segment on the short arm of chromosome 4. (F) Copy number probes at the SLC34A2 locus determined by an SNP array 6.0, the SLC34A2 genomic structure and the scale that starts from the telomere of the short arm of chromosome 4 are shown aligned. $\log _{2}$ ratio indicates the copy number of chromosomes at each position, ie, 0 (nulliploid), 1 (haploid), 2 (duploid) or 4 (tetraploid). $\log _{2}$ ratio of the probe CN_1062321 is 0 (nulliploid) while the copy number of the adjacent probes CN_1062320 and CN_1062322 is 2 (diploid), suggesting the presence of a homozygous deletion at CN_1062321. PCR reactions that amplify each exon of SLC34A2 failed to amplify exons 2-6 (shown by $\times$ ) and confirm the presence of a homozygous deletion. The nucleotide sequences of other exons and their flanking sequences have been confirmed to be the wild-type sequences.

causative gene for PAM, since the autozygous segments often contain the recessive disease genes, the autozygous segments could have been analysed to search for the chromosomal aberrations that affect the function of some genes which might have led to the deletion at SLC34A2. This shows that the analysis with a single inbred patient may identify a causative gene. By January 2007, only 2000 out of 22000 human genes have been associated with a disease ${ }^{10}$ many of the recessive genes are considered to be unidentified, especially those that cause diseases with a low penetrance or that occur only in elderly persons. When a distinct disease is seen in an inbred patient, a whole genome SNP study may be worth trying. Data obtained from only a single patient may therefore provide a clue to the causative gene.
Acknowledgements: The authors thank the medical technologists of Omihachiman Community Medical Center, the Department of Clinical Medicine, for their excellent technical assistance in echocardiography and preparation of pathological specimens.

Funding: This work was supported in part by grants-in-aid from Comprehensive Research on Aging and Health from the Ministry of Health, Labour and Welfare, Japan.

Competing interests: None.

Patient consent: Obtained.

\section{REFERENCES}

1. Crow JF. Genetic notes. 8th ed. New York: Macmillan, 1983.

2. Clark AG. The size distribution of homozygous segments in the human genome. Am J Hum Genet 1999;65:1489-92.

3. Lander ES, Botstein D. Homozygosity mapping: a way to map human recessive traits with the DNA of inbred children. Science 1987;236:1567-70. 
4. Wood CG, Valente EM, Bond J, et al. A new method for autozygosity mapping using single nucleotide polymorphisms (SNPs) and ExcludeAR. J Med Genet 2004;41:e101-4.

5. Afffymetrix. Affymetrix Genome-Wide Human SNP Array 6.0 Data Sheet.

6. Mariotta S, Ricci A, Papale M, et al. Pulmonary alveolar microlithiasis: report on $\mathbf{5 7 6}$ cases published in the literature. Sarcoid Vasc Diffuse Lung Dis 2004;21:173-81.

7. Tachibana T, Hayashi S, Jokoh $T$, et al. Diagnosis and management of pulmonary alveolar microlithiasis. Sarcoid Vasc Diffuse Lung Dis 2001;18(Suppl 1):58.
8. Corut A, Senyigit A, Ugur SA, et al. Mutations in SLC34A2 cause pulmonary alveolar microlithiasis and are possibly associated with testicular microlithiasis. Am J Hum Genet 2006;79:650-6.

9. Huqun, Izumi S, Miyazawa $\mathrm{H}$, et al. Mutations in the SLC34A2 gene are associated with pulmonary alveolar microlithiasis. Am J Respir Crit Care Med 2007;175:263-8.

10. McKusick VA. Mendelian inheritance in man and its online version, OMIM. Am J Hum Genet 2007; 80:588.

\section{Procalcitonin-guided antibiotic use for respiratory tract infections}

Antibiotic therapy is widely used in primary care to treat acute respiratory tract infections in spite of predominant viral aetiology. This study investigated whether procalcitonin (PCT), a biochemical marker elevated in systemic bacterial infections, can be used to guide antibiotic treatment.

A total of 458 adults with respiratory tract infection symptoms who, in their physician's opinion required antibiotics, were included. Patients were randomised to PCT-guided or standard antibiotic therapy. Antibiotic use in the PCT-guided therapy arm was related to raised serum levels. Follow-up data were collected at 7, 14 and 28 days after presentation. The primary outcome measure was the number of days of patient inactivity. Secondary outcome measures included antibiotic prescription rates and duration of treatment.

There was no difference in the number of days with restricted activities between the two groups. A 40\% reduction in antibiotic prescriptions was found in the PCT-guided group in those patients with an acute exacerbation of COPD, asthma or community acquired pneumonia, and an $80 \%$ reduction in those with upper respiratory tract infections or acute bronchitis. Interestingly, $15 \%$ of patients received antibiotics in the PCT group despite PCT levels being below the cut-off level.

The authors concluded that PCT-guided treatment did not compromise patient outcome in acute respiratory tract infections. However, patients were not blinded, possibly leading to bias in those who did not receive antibiotics. PCT could be used in the future to guide treatment and to reduce antibiotic use which, in turn, may help to decrease antibiotic resistance. However, changing physicians' methods of practice may prove a trickier task.

- Briel M, Schuetz P, Mueller B, et al. Procalcitonin-guided antibiotic use vs a standard approach for acute respiratory tract infections in primary care. Arch Intern Med 2008;168:2000-7.

\section{Flatley}

Correspondence to: Dr M Flatley, CT1 Senior House Officer, Royal Blackburn Hospital, Blackburn, Lancashire, UK; michaelflatley_2000@yahoo.com 